\title{
Effect of the chromo-electromagnetic field fluctuations on heavy quark propagation in a deconfined hadronic medium at energies available at the CERN Large Hadron Collider
}

\author{
Ashik Ikbal Sheikh and Zubayer Ahammed* \\ Variable Energy Cyclotron Centre, HBNI, 1/AF Bidhannagar, Kolkata 700 064, India \\ Prashant Shukla \\ Nuclear Physics Division, Bhabha Atomic Research Centre, HBNI, Mumbai 400085, India \\ Munshi G. Mustafa \\ Theory Division, Saha Institute of Nuclear Physics, HBNI, 1/AF Bidhannagar, Kolkata 700 064, India
}

(Received 21 March 2018; revised manuscript received 25 June 2018; published 21 September 2018)

\begin{abstract}
We consider the effect of the chromo-electromagnetic field fluctuations in addition to the collisional as well as the radiative energy losses suffered by heavy quarks while propagating through the hot and dense deconfined medium of quarks and gluons created in relativistic heavy ion collisions. The chromo-electromagnetic field fluctuations play an important role as they lead to an energy gain of heavy quarks of all momenta and are significant at low momentum. We include the effect of these fluctuations, for the first time, while computing the nuclear modification factor $\left(R_{A A}\right)$ of heavy mesons, viz., $D$ mesons and $B$ mesons, and compare with the experimental measurements in Pb-Pb collisions at $\sqrt{s_{N N}}=2.76$ and $5.02 \mathrm{TeV}$ by the CMS and ALICE experiments at the CERN Large Hadron Collider. Our results are found to be in very good agreement with the measurements.
\end{abstract}

DOI: 10.1103/PhysRevC.98.034915

\section{INTRODUCTION}

The main goal of high energy heavy-ion collisions at the Relativistic Heavy Ion Collider (RHIC) at BNL and the Large Hadron Collider (LHC) at CERN is to produce a hot and dense deconfined state of QCD matter, called quark-gluon plasma (QGP). Many experimental results indicate that this new deconfined state of matter has been formed during high energy heavy-ion collisions at RHIC [1] and LHC [2]. One of the features of this deconfined state created in heavy ion collisions is the suppression of high energy hadrons compare to the case in $p-p$ collisions, which is called jet quenching. The jet quenching is caused by the energy loss of initial hard partons via collisional and radiative energy loss inside the deconfined medium. Jet quenching was first anticipated by Bjorken [3] as a crucial probe of the deconfined medium. The energy loss suffered by highly energetic partons, both light and heavy quarks, in the deconfined QCD medium is of immense interest because it unravels the dynamical properties of the medium. The study of energy loss of heavy quarks in

*za@vecc.gov.in

Published by the American Physical Society under the terms of the Creative Commons Attribution 4.0 International license. Further distribution of this work must maintain attribution to the author(s) and the published article's title, journal citation, and DOI. Funded by $S C O A P^{3}$. the deconfined medium has been an ever active and important field of research [4-33].

Heavy quarks are mostly produced in the early stage of heavy ion collisions from the initial fusion of partons. They may also be produced in the QGP, if the initial temperature of the QGP is high enough compared to the mass of the heavy quark. However, the probability of production of heavy quarks is usually small in the QGP phase and almost zero in the hadronic phase. Hence, the total number of heavy quarks becomes frozen at a very early stage in the history of the collisions, which makes them a good probe of the QGP. The heavy quarks, immediately after their production, propagate through the hot and dense medium and start losing energy during their path of travel. The energy loss suffered by the heavy quarks is reflected in the transverse momentum spectra and nuclear modification factor of heavy mesons. Heavy quarks lose energy mainly by two different mechanisms in the QGP: elastic collisions with the light partons inside the QGP and by radiating gluons, as in the bremsstrahlung process.

The energy loss formalisms generally treat the medium properties in an average manner by ignoring the fluctuations in the QGP. The QGP is a statistical ensemble of mobile colored charged particles, which can be characterized by omnipresent stochastic fluctuations. These microscopic fluctuations generally couple to the external perturbations and would affect the response of the medium. The effect of electromagnetic field fluctuations during the passage of charged particles though a nonrelativistic classical plasma has been calculated by several authors [34-39]. When an energetic parton (either light or 
heavy) propagates in the QGP, a retarding force acts on the parton in the plasma due to the chromo-electric field generated by the parton itself while moving. The energy loss of the parton is obtained through the work done by this retarding force that acts on the parton and is determined within the linear response theory in terms of the dielectric tensor of the medium. Nevertheless this approach does not take into account the chromo-electric field fluctuations and the particle recoil in the plasma. To include these effects one needs to perform two kind of averaging [30]: (a) an ensemble average with respect to the equilibrium density matrix and (b) a time average over random fluctuations in the plasma. This averaging is applicable to high energy jets of both the light and heavy quarks in the QGP. The effect of parton energy loss due to stimulated gluon emission and thermal absorption has been reported in Ref. [40]. On the other hand the effect of chromoelectromagnetic fluctuations in the QGP leads to energy gain of heavy quarks of all momenta but is more pronounced for the lower momentum ones [30]. This is because the moving parton in the QGP encounters a statistical change in the energy due to the fluctuations of the chromo-electromagnetic fields and the velocity of the particle under the influence of this field. The effects of such fluctuations were not considered in earlier literature studying the hadron spectra in heavy ion collisions.

In this article, we investigate for the first time the effect of the chromo-electromagnetic field fluctuations leading to energy gain of heavy quarks in addition to the collisional and the radiative energy loss on the nuclear modification factors for $D$ and $B$ mesons, and we compare with the measurements of both ALICE and CMS experiments in $\mathrm{Pb}-\mathrm{Pb}$ collisions at $\sqrt{s_{N N}}=2.76 \mathrm{TeV}$ and CMS measurements at $\sqrt{s_{N N}}=$ $5.02 \mathrm{TeV}$.

The paper is organized as follows: In Sec. II, we briefly outline the basic formalism containing heavy quark production and fragmentation, models for both collisional and radiative energy loss, and energy gain due to field fluctuations, medium evolution, and initial conditions. We consider the collisional energy loss of heavy quarks by the Peigne and Pashier (PP) formalism [19] and the radiative energy loss by the Abir, Jamil, Mustafa, and Srivastava (AJMS) formalism [31] along with the energy gained due to the chromo-electric field fluctuations prescription by Chakraborty, Mustafa, and Thoma (CMT) in Ref. [30]. In Sec. III we discuss our results, and the conclusion is given in Sec. IV.

\section{METHODOLOGY}

\section{A. Heavy quark production and fragmentation}

The heavy quarks in $p$ - $p$ collisions are mainly produced by fusion of gluons or light quarks [41]. Their production cross sections in $p$ - $p$ collisions are obtained using factorization with elementary cross sections calculated up to leading order (LO) and next-to-leading order (NLO) C10 parton density functions [42]. For heavy ion collisions, the shadowing effect is taken into account by using the spatially dependent EPS09 [43] sets. The differential cross section, including the nuclear shadowing effect corresponding to a given centrality class between impact parameters $b_{1}$ and $b_{2}$, is calculated. Now the spectrum in $\mathrm{Pb}-\mathrm{Pb}$ collisions can be obtained by shifting the calculated differential cross section by the momentum loss $\Delta p_{T}$. For fragmentation of $c$ quarks into $D$ mesons and $b$ quarks into $B$ mesons, the Peterson fragmentation function [44], with parameters $\epsilon_{c}=0.016$ for $c$ quarks and $\epsilon_{b}=0.0012$ for $b$ quarks, is used. For other details of the production and fragmentation of heavy quarks we refer the readers to Ref. [33].

Finally, the nuclear modification factor $R_{A A}$ is computed as

$$
R_{A A}\left(p_{T}, b_{1}, b_{2}\right)=\frac{\frac{d^{2} \sigma_{P b P b}\left(p_{T}, b_{1}, b_{2}\right)}{d p_{T}^{2} d y}}{\int_{b 1}^{b 2} d^{2} b T_{A A} \frac{d^{2} \sigma_{p p}\left(p_{T}\right)}{d p_{T}^{2} d y}}
$$

where $b_{1}$ and $b_{2}$ are the impact parameters corresponding to a given centrality of collision and $T_{A A}$ is the nuclear overlap function.

\section{B. Medium evolution and initial condition}

As a heavy quark loses energy during its passage through the QGP medium, one needs to estimate the path length it is traversing inside the medium. We consider a heavy quark that is produced at a point $(r, \phi)$ in a heavy ion collision and propagates at an angle $\phi$ with respect to $\hat{r}$ in the transverse plane. So the path length $L$ covered by the heavy quark inside the medium is given by [45]

$$
L(r, \phi)=\sqrt{R^{2}-r^{2} \sin ^{2} \phi}-r \cos \phi,
$$

where $R$ is the radius of the colliding nuclei. The average distance traveled by the heavy quark inside the plasma is

$$
\langle L\rangle=\frac{\int_{0}^{R} r d r \int_{0}^{2 \pi} L(r, \phi) T_{A A}(r, b) d \phi}{\int_{0}^{R} r d r \int_{0}^{2 \pi} T_{A A}(r, b) d \phi},
$$

where the nuclear overlap function, $T_{A A}(r, b)$, at an impact parameter $b$ is averaged over given centrality class. The effective path length of a heavy quark having transverse mass $m_{T}$ and transverse momentum $p_{T}$ in the QGP of life time $\tau_{f}$ is obtained as

$$
L_{\mathrm{eff}}=\min \left[\langle L\rangle, \frac{p_{T}}{m_{T}} \times \tau_{f}\right] .
$$

The evolution of the system for each centrality bin is assumed to be governed by an isentropic cylindrical expansion as described in Ref. [46,47]. The entropy conservation condition $s(T) V(\tau)=s\left(T_{0}\right) V\left(\tau_{0}\right)$ is used to obtain the temperature as a function of proper time. The equation of state obtained by lattice QCD and the hadronic resonance gas formulation has been used. The initial volume corresponding to a given centrality is obtained by $V\left(\tau_{0}\right)=\pi\left[R_{t r}\left(N_{\text {part }}\right)\right]^{2} \tau_{0}$. The transverse size $R_{t r}\left(N_{\text {part }}\right)$ for a given centrality with number of participants $\left(N_{\text {part }}\right)$ is obtained as $R_{t r}\left(N_{\text {part }}\right)=R \sqrt{2 A / N_{\text {part }}}$, where $A$ is the mass number of the colliding nucleus [47].

The energy loss as a function of proper time is calculated, which is then averaged over the temperature evolution for each centrality bin. The initial and freeze-out times are taken as $\tau_{0}=0.3 \mathrm{fm}$ and $\tau_{f}=6 \mathrm{fm}$, respectively, as used in Ref. [33]. 
TABLE I. Parameters for medium evolution.

\begin{tabular}{lccc}
\hline \hline$\sqrt{s_{N N}}(\mathrm{TeV})$ & 2.76 & 2.76 & 5.02 \\
Centrality class $(\%)$ & $0-10$ & $0-100$ & $0-100$ \\
$\langle b\rangle(\mathrm{fm})$ & 3.44 & 9.68 & 9.65 \\
$N_{\text {part }}$ & 356 & 113 & 114 \\
$\langle L\rangle(\mathrm{fm})$ & 5.73 & 4.16 & 4.18 \\
$T_{0}(\mathrm{GeV})$ & 0.467 & 0.436 & 0.469 \\
\hline \hline
\end{tabular}

Various parameters used in our calculations for different centrality classes such as impact parameter $\langle b\rangle, N_{\text {part }},\langle L\rangle$, and initial temperature $T_{0}$ are given in Table I.

\section{Collisional energy loss: Peigne and Peshier (PP) formalism}

One of the important mechanisms in which heavy quarks may lose energy inside the QGP is through elastic collisions. The calculation of collisional energy loss per unit length $d E / d x$ has been reported in the past by several authors $[4,5,48]$. The most detailed calculation of $d E / d x$ was made by Brateen and Thoma [5], and was based on their previous QED calculation of $d E / d x$ for muons [49]. This calculation of Brateen and Thoma for $d E / d x$ is based on an assumption that the momentum exchange $q$ in elastic collisions is much smaller than the energy of the heavy quark, $E$, i.e., $q \ll E$. But this is not an appropriate assumption in the domain $E \gg$ $M^{2} / T$, where $M$ is the mass of the heavy quark and $T$ is the temperature of the medium. The improved differential energy loss expression, valid for $E \gg M^{2} / T$, has been obtained using Peigne and Pashier [19] as

$$
\begin{aligned}
\frac{d E}{d x}= & \frac{4 \pi \alpha_{s}^{2} T^{2}}{3}\left[\left(1+\frac{n_{f}}{6}\right) \ln \left(\frac{E T}{\mu_{g}^{2}}\right)+\frac{2}{9} \ln \left(\frac{E T}{M^{2}}\right)\right] \\
& +\frac{4 \pi \alpha_{s}^{2} T^{2}}{3} c\left(n_{f}\right)
\end{aligned}
$$

where $\mu_{g}^{2}=4 \pi \alpha_{s} T^{2}\left(1+n_{f} / 6\right)$ is the square of Debye screening mass, $n_{f}$ is the number of active quark flavors, $c\left(n_{f}\right) \approx 0.146 n_{f}+0.05$, and $\alpha_{s}=0.3$ is the strong coupling constant.

\section{Radiative energy loss: Abir, Jamil, Mustafa, and Srivastava (AJMS) formalism}

The most important and dominant way of energy loss from a fast parton inside the QGP is due to gluon radiation. The first attempt to estimate the radiative energy loss was made in Ref. [6]. Later many authors [8,9,12,15-18,22,24,25,31] also estimated the energy loss with various ingredients and kinematical conditions. In Refs. [8,9] the soft gluon emission from heavy quarks was estimated and was found to be suppressed as compared to the case of the light quarks due to the mass effect, known as the dead cone effect. The radiative energy loss induced by the medium due to the dead cone effect was limited only to the forward direction. In Ref. [12], some of the constraints imposed in the work of Refs. [8,9] (e.g., the gluon emission angle and the scaled mass of the heavy quark with its energy) were relaxed and a generalized dead cone was obtained which led to a very compact expression for the gluon emission probability off a heavy quark. Based on the generalized dead cone approach and the gluon emission probability [12], AJMS [31] computed the heavy quark radiative energy $\operatorname{loss}^{1}$ as

$$
\begin{aligned}
\frac{d E}{d x}= & 24 \alpha_{s}^{3} \rho_{Q G P} \frac{1}{\mu_{g}}\left(1-\beta_{1}\right) \\
& \times\left(\sqrt{\frac{1}{\left(1-\beta_{1}\right)} \ln \left(\frac{1}{\beta_{1}}\right)}-1\right) \mathcal{F}(\delta),
\end{aligned}
$$

with

$$
\begin{aligned}
\mathcal{F}(\delta)= & 2 \delta-\frac{1}{2} \ln \left(\frac{1+\frac{M^{2}}{s} e^{2 \delta}}{1+\frac{M^{2}}{s} e^{-2 \delta}}\right) \\
& -\left(\frac{\frac{M^{2}}{s} \sinh (2 \delta)}{1+2 \frac{M^{2}}{s} \cosh (2 \delta)+\frac{M^{4}}{s^{2}}}\right),
\end{aligned}
$$

where

$$
\delta=\frac{1}{2} \ln \left[\frac{1}{\left(1-\beta_{1}\right)} \ln \left(\frac{1}{\beta_{1}}\right)\left(1+\sqrt{1-\frac{\left(1-\beta_{1}\right)}{\ln \frac{1}{\beta_{1}}}}\right)^{2}\right],
$$

and $\rho_{Q G P}$ is the density of the QGP medium which acts as a background containing the target partons. If $\rho_{q}$ and $\rho_{g}$ are the densities of quarks and gluons respectively in the medium, then $\rho_{Q G P}$ is given by

$$
\begin{aligned}
\rho_{Q G P} & =\rho_{q}+\frac{9}{4} \rho_{g} \\
\beta_{1} & =\frac{\mu_{g}^{2}}{C E T} \\
C & =\frac{3}{2}-\frac{M^{2}}{4 E T}+\frac{M^{4}}{48 E^{2} T^{2} \beta_{0}} \ln \left(\frac{M^{2}+6 E T\left(1+\beta_{0}\right)}{M^{2}+6 E T\left(1-\beta_{0}\right)}\right) \\
\beta_{0} & =\sqrt{1-\frac{M^{2}}{E^{2}}}
\end{aligned}
$$

\section{E. Energy gain by chromo-electromagnetic field fluctuations: Chakraborty, Mustafa, and Thoma (CMT) Formalism}

The energy loss calculations, both collisional and radiativ,e of heavy quarks in the QGP were obtained by treating the QGP medium without considering microscopic fluctuations. However, QGP, being a statistical system, is characterized by stochastic chromo-electromagnetic field fluctuations. A quantitative estimate of the effect of the microscopic electromagnetic fluctuations on the propagation of a heavy quark was done using the semiclassical approximation. This led to an energy gain of the heavy quark caused by the statistical change in the energy of the moving parton in the QGP due to the

${ }^{1}$ Later a kinematical correction was made in Ref. [32]. 


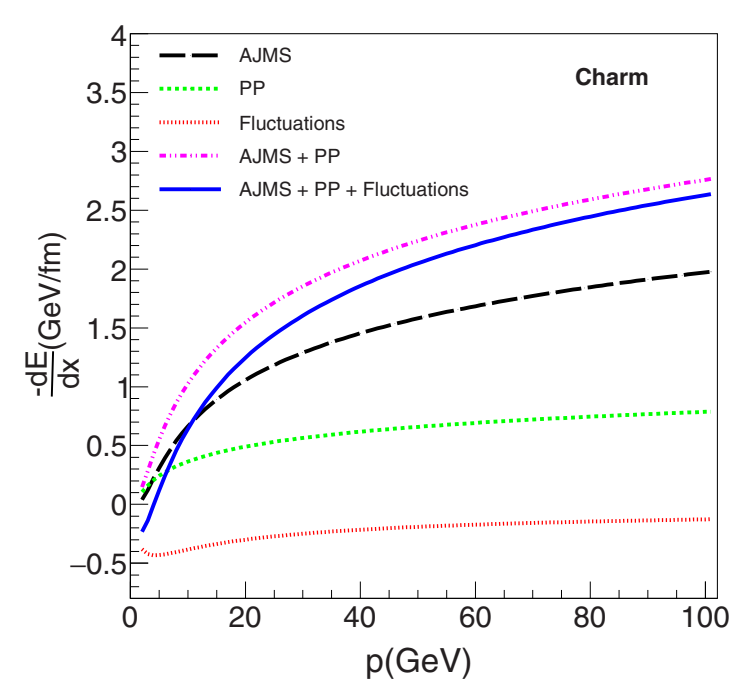

FIG. 1. The energy loss of a charm quark inside the QGP medium as a function of its momentum, obtained using PP [19], AJMS [31], and fluctuation [30] methods.

fluctuations of the chromo-electromagnetic fields as well as the velocity of the particle under the influence of this field. The leading-log (LL) contribution of the energy gain was obtained [30] as

$$
\begin{aligned}
\left(\frac{d E}{d x}\right)_{\mathrm{fl}}^{\mathrm{LL}}= & 2 \pi C_{F} \alpha_{s}^{2}\left(1+\frac{n_{f}}{6}\right) \frac{T^{3}}{E v^{2}} \ln \frac{1+v}{1-v} \\
& \times \ln \frac{k_{\max }}{k_{\min }}
\end{aligned}
$$

where $k_{\min }=\mu_{g}$ is the Debye mass and $k_{\max }=$ $\min \left[E, 2 q(E+p) / \sqrt{M^{2}+2 q(E+p)}\right]$, with $q \sim T$, is the typical momentum of the thermal partons. One can physically interpret the energy gain of a heavy quark as being due to the absorption of gluons during its propagation.

In Figs. 1 and 2, we display the various contributions to the differential energy loss of charm and bottom quarks, respectively. Also the differential contribution due to the effect of field fluctuations is included in both figures. Our choices of parameters are $n_{f}=2, \alpha_{s}=0.3$, charm quark mass $M_{c}=$ $1.25 \mathrm{GeV}$, and bottom quark mass $M_{b}=4.2 \mathrm{GeV}$. It is observed that the energy loss increases as momentum of quarks increases, but the rate of increment is higher in the lower momentum region. It is also seen that the AJMS differential radiative energy loss always dominates over PP collisional energy loss for charm quarks, whereas for bottom quarks the differential PP collisional energy loss dominates over the AJMS differential radiative energy loss up to a momentum of $10 \mathrm{GeV}$, beyond which the AJMS radiative energy loss takes over for charm quarks as compared to bottom quarks. The differential energy loss $(-d E / d x)$ is negative due to the field fluctuations, which implies energy gain due to the absorption of gluons by heavy quarks. This energy gain due to the field fluctuations is found to be significant in the momentum range $4-20 \mathrm{GeV}$. This energy gain is relatively more for charm quarks compared to bottom quarks.

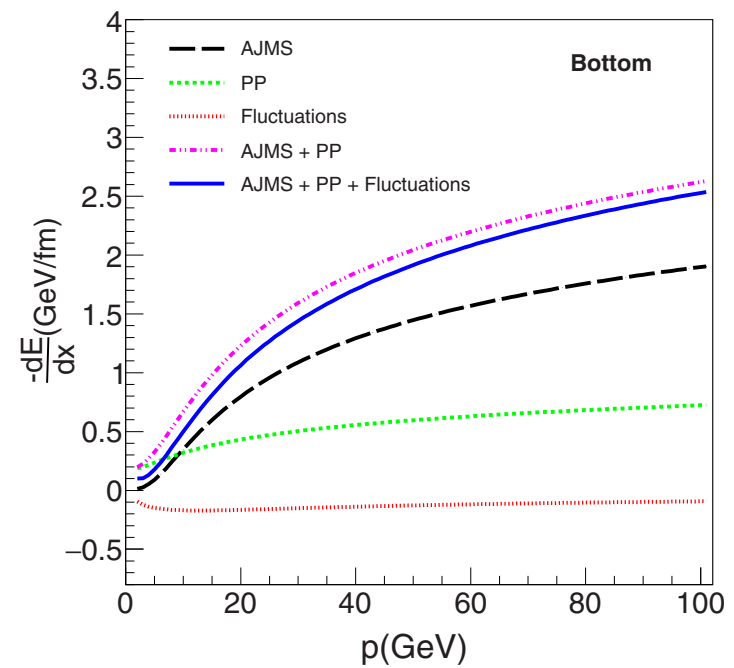

FIG. 2. The energy loss of a bottom quark inside the QGP medium as a function of its momentum, obtained using PP [19], AJMS [31], and fluctuation [30] methods.

Figures 3 and 4 display the fractional energy loss from collisional and radiative processes, and also the energy gain due to the field fluctuations for charm and bottom quarks, respectively. It is clear from both the figures that the energy gain for heavy quarks is relatively more in the lower momentum region (4-40 MeV) than in the higher momentum ( $>40$ $\mathrm{MeV}$ ) region. This means that the field fluctuations and thus the energy gain become substantial only in the low velocity limit of heavy quarks. The effect of these field fluctuations is to reduce the total energy loss of heavy quarks up to a certain value of momentum, beyond which their contributions gradually diminish.

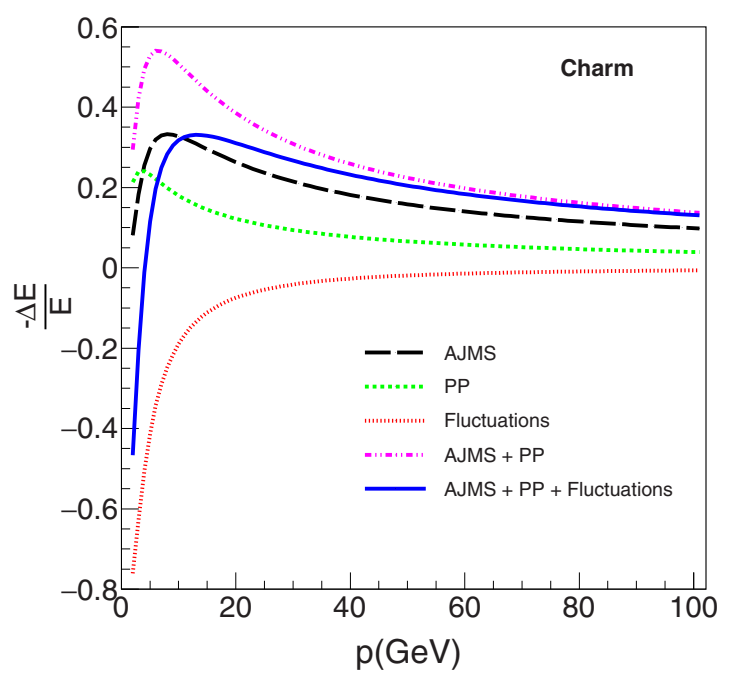

FIG. 3. Fractional energy loss of a charm quark inside the QGP due to fluctuations, collisions (PP), and radiation (AJMS) as a function of its momentum. The path length considered here is $L=5 \mathrm{fm}$. 


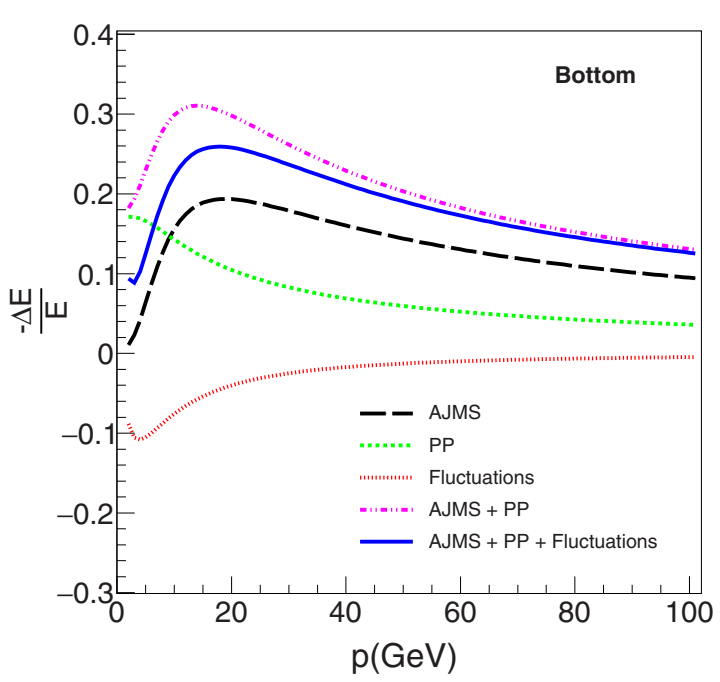

FIG. 4. Fractional energy loss of a bottom quark inside the QGP due to fluctuations, collisions (PP), and radiation (AJMS) as a function of its momentum. The path length considered here is $L=5 \mathrm{fm}$.

\section{RESULTS AND DISCUSSIONS}

Figures 5 and 6 display the nuclear modification factor $R_{A A}$ as a function of $p_{T}$ for a $D^{0}$ meson in $\mathrm{Pb}-\mathrm{Pb}$ collisions for $(0-10) \%$ and $(0-100) \%$ centralities, respectively, considering both collisional and radiative energy loss along with the energy gain due to the field fluctuations. The results are also compared with ALICE [50] and CMS data [51].

We observe that only radiative energy loss (AJMS) or the collisional energy loss (PP) along with the radiative energy

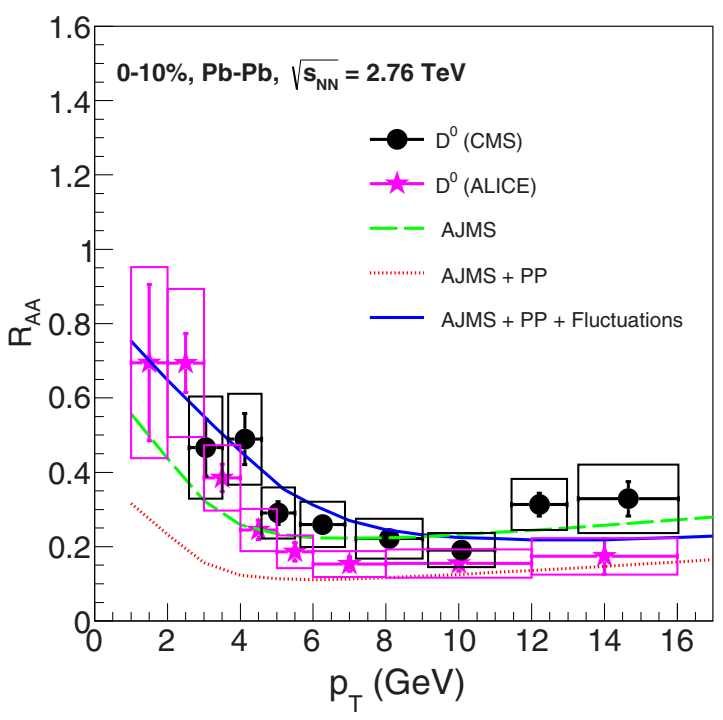

FIG. 5. The nuclear modification factor $R_{A A}$ of $D^{0}$ mesons with collisional (PP) and radiative (AJMS) energy loss along with the effect of fluctuations as a function of transverse momentum $p_{T}$ for $(0-10) \%$ centrality in $\mathrm{Pb}-\mathrm{Pb}$ collisions at $\sqrt{s_{N N}}=2.76 \mathrm{TeV}$. The experimental data are taken from measurements by the ALICE [50] and CMS experiments [51].

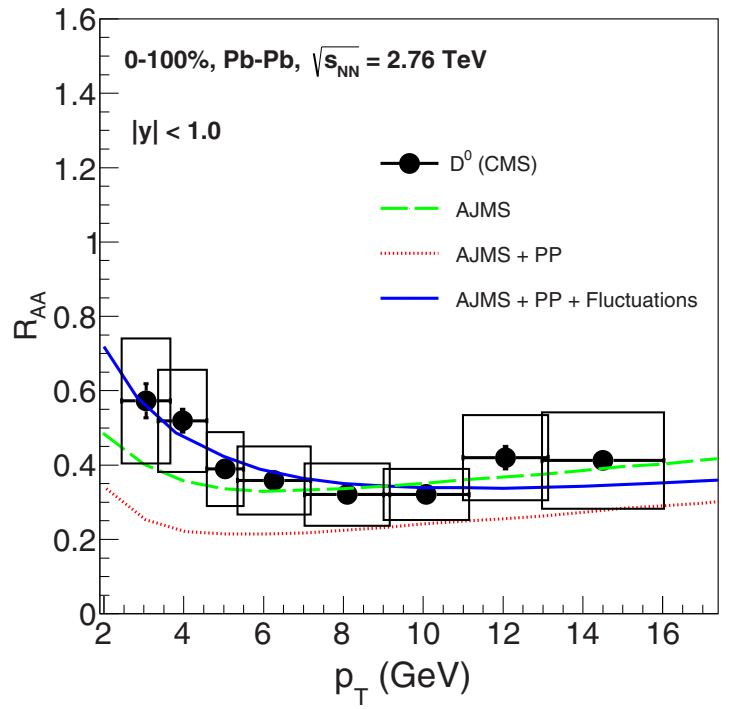

FIG. 6. The nuclear modification factor $R_{A A}$ of $D^{0}$ mesons with collisional (PP) and radiative (AJMS) energy loss along with the effect of fluctuations as a function of transverse momentum $p_{T}$ for $(0-100) \%$ centrality in $\mathrm{Pb}-\mathrm{Pb}$ collisions at $\sqrt{s_{N N}}=2.76 \mathrm{TeV}$. The experimental data are taken from measurements by the CMS experiment [51].

loss cannot explain the data properly. As can be seen, the radiative loss along with the collisional energy loss overestimates the data in entire $p_{T}$ range whereas only the radiative one alone can describe the data for the transverse momentum $p_{T}>10 \mathrm{GeV}$. This is because the radiative energy loss has a dominant contribution compared to the collisional one. When the energy gain due to the chromo-electromagnetic field fluctuations are included in addition to both the collisional and the radiative losses the measured data are well described in the entire $p_{T}$ range. We emphasize that the chromo-electromagnetic field fluctuations are found to play an important role in the propagation of the heavy quark jets in a QGP vis-à-vis the nuclear modification factor of heavy flavored hadrons.

Figure 7 displays the nuclear modification factor $R_{A A}$ of $D^{0}$ mesons as a function of $p_{T}$, for $(0-100) \%$ centrality in $\mathrm{Pb}$ $\mathrm{Pb}$ collisions at $\sqrt{s_{N N}}=5.02 \mathrm{TeV}$, obtained using collisional (PP) energy loss, radiative (AJMS) energy loss, and the effect of field fluctuations in $\mathrm{Pb}-\mathrm{Pb}$ collisions at $\sqrt{s_{N N}}=5.02 \mathrm{TeV}$. The experimental data are taken from the CMS Collaboration [52]. Again the radiative energy loss (AJMS) alone can describe the data above transverse momentum $10 \mathrm{GeV}$ but the $R_{A A}$ spectra in the full $p_{T}$ range can be described when the effect of fluctuations is taken into consideration.

In Fig. 8 the nuclear modification factor $R_{A A}$ for $B^{+}$ mesons in $\mathrm{Pb}-\mathrm{Pb}$ collisions at $\sqrt{s_{N N}}=5.02 \mathrm{TeV}$ is displayed considering both collisional and radiative energy loss along with the effect of the field fluctuations, and is compared with CMS data [53]. The radiative energy loss itself produces a small suppression, but when the collisional one is added it gives more suppression than the suppression measured by the CMS experiment. Importantly, with the inclusion of the energy gain due to the chromo-electromagnetic field fluctuations in addition to both the radiative and collisional losses, the 


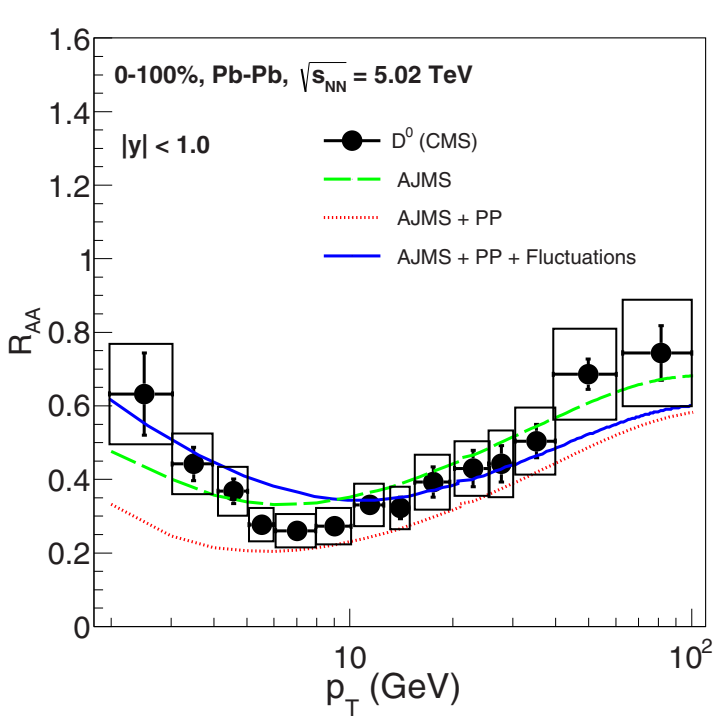

FIG. 7. The nuclear modification factor $R_{A A}$ of $D^{0}$ mesons with collisional (PP) and radiative (AJMS) energy loss along with the effect of field fluctuations as a function of transverse momentum $p_{T}$ for $(0-100) \%$ centrality in $\mathrm{Pb}-\mathrm{Pb}$ collisions at $\sqrt{s_{N N}}=5.02 \mathrm{TeV}$. The experimental data are taken from measurements by the CMS experiment [52].

suppression is found to be very close to the measured data within their uncertainties.

We would like to mention that theoretical uncertainties may appear due to following reasons:

(i) The thermalization of hot and dense matter is an unsettled issue which causes an uncertainty in the initial conditions. The initial time, $\tau_{0}$, may vary between 0.1 and $0.5 \mathrm{fm} / c$, which effectively causes variation in the initial temperature $T_{0}$ and thus in the effective temperature of the medium.

(ii) The semiclassical approximation has been used to calculate the mean energy loss, and has been shown to be equivalent to the hard thermal loop approximation based on the weak coupling limit $[4,5,19,31]$. It also corresponds to neglecting the non-Abelian terms in the QCD equations of motion.

(iii) We assume a constant momentum and temperatureindependent coupling constant instead of running coupling.

(iv) The parton distribution and fragmentation function involve uncertainties which in turn would affect the $R_{A A}$.

\section{CONCLUSION}

The energy loss encountered by an energetic parton in a QGP medium reveals the dynamical properties of that medium

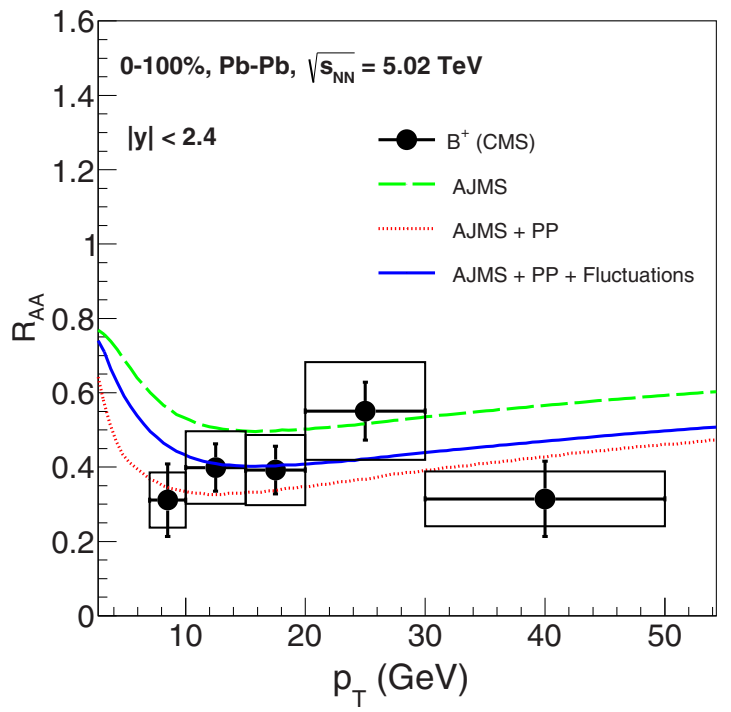

FIG. 8. The nuclear modification factor $R_{A A}$ of $B^{+}$mesons with collisional (PP) and radiative (AJMS) energy loss along with the effect of field fluctuations as a function of transverse momentum $p_{T}$ for $(0-100) \%$ centrality in $\mathrm{Pb}-\mathrm{Pb}$ collisions at $\sqrt{s_{N N}}=5.02 \mathrm{TeV}$. The experimental data are taken from measurements by the CMS experiment [53].

in view of jet quenching of high energy partons. This is usually reflected in the transverse momentum spectra and nuclear modification factor of mesons which are measured in heavy ion experiments. For the phenomenology of heavy quark jet quenching, the field fluctuations in the QGP medium were not considered in the literature before. In this article, for the first time, we have considered during the propagation of high energy heavy quarks the energy gain due to field fluctuations along with the energy loss caused by the collisions and gluon radiations inside the QGP medium. The nuclear modification factors $R_{A A}$ for $D$ mesons and $B$ mesons in $\mathrm{Pb}-\mathrm{Pb}$ collisions at $\sqrt{s_{N N}}=2.76$ and $5.02 \mathrm{TeV}$ are calculated by including both the types of energy losses and the field fluctuation effect. We note that the radiative energy loss alone can describe the $D$-meson suppressions at higher transverse momentum. Nevertheless, the nuclear modification factors for both $D$ and $B$ mesons are found to agree quite well with those data in the entire $p_{T}$ range measured by CMS and ALICE experiments at LHC energies, if the energy gain due to the field fluctuations is taken into account in addition to the collisional and radiative loss in the medium. The effect of field fluctuations in hot and dense QGP medium is found to play an important role in the propagation of heavy quarks also in describing the experimental data for heavy quark quenching.

\section{ACKNOWLEDGMENTS}

The authors would like to acknowledge discussions with Kapil Saraswat and Subhasis Chattopadhyay.
[1] M. Gyulassy and L. McLerran, Nucl. Phys. A 750, 30 (2005).
[2] J. W. Harris and B. Muller, Ann. Rev. Nucl. Part. Sci. 46, 71 (1996). 
[3] J. D. Bjorken, Fermilab Report No, PUB-82/59-THY, 1982 (unpublished).

[4] M. H. Thoma and M. Gyulassy, Nucl. Phys. B 351, 491 (1991).

[5] E. Braaten and M. H. Thoma, Phys. Rev. D 44, R2625(R) (1991).

[6] M. G. Mustafa, D. Pal, D. K. Srivastava, and M. H. Thoma, Phys. Lett. B 428, 234 (1998); 438, 450(E) (1998).

[7] M. G. Mustafa, Phys. Rev. C 72, 014905 (2005); M. G. Mustafa, D. Pal, and D. K. Srivastava, ibid. 57, 889 (1998).

[8] Y. L. Dokshitzer and D. E. Kharzeev, Phys. Lett. B 519, 199 (2001).

[9] Y. L. Dokshitzer, V. A. Khoze, and S. I. Troian, J. Phys. G 17, 1602 (1991).

[10] O. Fochler, Z. Xu, and C. Greiner, Phys. Rev. C 82, 024907 (2010).

[11] S. K. Das, J.-E. Alam, and P. Mohanty, Phys. Rev. C 82, 014908 (2010); 80, 054916 (2009).

[12] R. Abir, C. Greiner, M. Martinez, M. G. Mustafa, and J. Uphoff, Phys. Rev. D 85, 054012 (2012).

[13] R. Abir, C. Greiner, M. Martinez, and M. G. Mustafa, Phys. Rev. D 83, 011501(R) (2011).

[14] S. K. Das and J.-E. Alam, Phys. Rev. D 82, 051502(R) (2010); T. Bhattacharyya, S. Mazumder, S. K. Das, and J.-E. Alam, ibid. 85, 034033 (2012).

[15] O. Fochler, Z. Xu, C. Greiner, Phys. Rev. Lett. 102, 202301 (2009).

[16] P. B. Gossiaux, J. Aichelin, T. Gousset, and V. Guiho, J. Phys. G 37, 094019 (2010).

[17] W. A. Horowitz, Nucl. Phys. A 855, 225 (2011).

[18] S. Wicks, W. Horowitz, M. Djordjevic, and M. Gyulassy, Nucl. Phys. A 784, 426 (2007); 783, 493 (2007); M. Djordjevic and M. Gyulassy, ibid. 733, 265 (2004).

[19] S. Peigne and A. Peshier, Phys. Rev. D 77, 114017 (2008).

[20] U. Jamil and D. K. Srivastava, J. Phys. G 37, 085106 (2010).

[21] N. Armesto, C. A. Salgado, and U. A. Wiedemann, Phys. Rev. D 69, 114003 (2004).

[22] N. Armesto, M. Cacciari, A. Dainese, C. A. Salgado, and U. A. Wiedemann, Phys. Lett. B 673, 362 (2006).

[23] B. Z. Kopeliovich, I. K. Potashnikova, and I. Schmidt, Phys. Rev. C 82, 037901 (2010).

[24] W. C. Xiang, H. T. Ding, D. C. Zhou, and D. Rohrich, E. Phys. J. A 25, 75 (2005).

[25] I. Vitev, J. Phys. G 35, 104011 (2008).

[26] M. Younus, C. E. Coleman-Smith, S. A. Bass, and D. K. Srivastava, Phys. Rev. C 91, 024912 (2015).

[27] S. K. Das, F. Scardina, S. Plumari, and V. Greco, Phys. Rev. C 90, 044901 (2014).
[28] J. Uphoff, O. Fochler, Z. Xu, and C. Greiner, Nucl. Phys. A 855, 444 (2011).

[29] J. Uphoff, O. Fochler, Z. Xu, and C. Greiner, Phys. Rev. C 84, 024908 (2011).

[30] P. Chakraborty, M. G. Mustafa, and M. H. Thoma, Phys. Rev. C 75, 064908 (2007).

[31] R. Abir, U. Jamil, M. G. Mustafa, and D. K. Srivastava, Phys. Lett. B 715, 183 (2012).

[32] K. Saraswat, P. Shukla, and V. Singh, Nucl. Phys. A 934, 83 (2015).

[33] K. Saraswat, P. Shukla, and V. Singh, Nucl. Phys. A 961, 169 (2017).

[34] S. Gasirowicz, M. Neumann, and R. J. Riddel, Phys. Rev. 101, 922 (1965).

[35] A. G. Sitenko, Electromagnetic Fluctuations in Plasma (Academic, New York, 1967).

[36] A. I. Akhiezer, I. A. Akhiezer, R. V. Polovin, A. G. Sitenko, and K. N. Stepanov, Plasma Electrodynamics (Pergamon, Oxford, 1975).

[37] G. Kalman and A. Ron, Ann. Phys. (NY) 16, 118 (1961).

[38] W. B. Thompson and J. Hubbard, Rev. Mod. Phys. 32, 714 (1960).

[39] S. Ichimaru, Basic Principles of Plasma Physics (Benjamin, Reading, 1973).

[40] E. Wang and X.-N. Wang, Phys. Rev. Lett. 87, 142301 (2001).

[41] B. L. Combridge, Nucl. Phys. B 151, 429 (1979).

[42] H.-L. Lai, M. Guzzi, J. Huston, Z. Li, P. M. Nodolsky, J. Pumplin, and C.-P. Yuan, Phys. Rev. D 82, 074024 (2010).

[43] K. J. Eskola, H. Paukkunen, and C. A. Salgado, J. High Energy Phys. 04 (2009) 065.

[44] C. Peterson, D. Schlatter, I. Schmitt, and P. M. Zerwas, Phys. Rev. D 27, 105 (1983).

[45] B. Muller, Phys. Rev. C 67, 061901 (2003).

[46] X. Zhao and R. Rapp, Nucl. Phys. A 859, 114 (2011).

[47] V. Kumar, P. Shukla, and R. Vogt, Phys. Rev. C 92, 024908 (2015).

[48] A. Meistrenko, A. Pashier, J. Uphoff, and C. Greiner, Nucl. Phys. A 901, 51 (2013).

[49] E. Braaten and M. H. Thoma, Phys. Rev. D 44, 1298 (1991).

[50] J. Adam et al. (ALICE Collaboration), J. High Energy Phys. 03 (2016) 081.

[51] CMS Collaboration, CERN Report No. CMS-PAS-HIN-15005, 2015 (unpubished).

[52] CMS Collaboration, Phys. Lett. B 782, 474 (2018).

[53] A. M. Sirunyan et al. (CMS Collaboration), Phys. Rev. Lett. 119, 152301 (2017). 